\title{
Modelling of Thermoelectric Generator with Heat Pipe Assist for Range Extender Application
}

\author{
Francisco P. Brito \\ Dpt. Mech. Engineering \\ University of Minho \\ Guimarães (Portugal) \\ francisco@dem.uminho.pt
}

\author{
Jorge Martins \\ Dpt. Mech. Engineering \\ University of Minho \\ Guimarães (Portugal) \\ jmartins@dem.uminho.pt
}

\author{
L.M. Goncalves \\ Dpt. Industrial Electronics \\ University of Minho \\ Guimarães (Portugal) \\ lgoncalves@dei.uminho.pt
}

\author{
Rui Sousa \\ Dpt. Industrial Electronics \\ University of Minho \\ Guimarães (Portugal) \\ a48008@alunos.uminho.pt
}

\begin{abstract}
Recent trends towards electrification of vehicles favour the adoption of waste energy recovery into electricity. Batteryonly Electric Vehicles (BEV) need a very large energy storage system so the use of a Range Extender (RE) may allow a significant downsizing of these bulky components. The Internal Combustion Engines (ICE) have two major discarded energy fluxes, engine cooling and exhaust gas. In Extended Range Electric Vehicles (EREV) and hybrids the potential for heat conversion into electricity is particularly convenient. The direct conversion of thermal energy into electricity, using Thermoelectric Generators (TEG) is very attractive in terms of complexity. However, current commercial TEG modules based on Seebeck effect are temperature limited, so they are unable to be in direct contact with the exhaust gases. A way to downgrade the temperature levels without reducing its potential is to interpose Heat Pipes (HP) between the exhaust gas and the modules. This control of maximum temperature at the modules is achieved by regulating the pressure of phase change of the HP fluid. Such design is convenient for engines with large thermal load variations, such as the RE being developed by the team, with a low $(15 \mathrm{~kW})$ and a high $(40 \mathrm{~kW})$ power mode of operation. This system will be able to operate efficiently in both modes. The present work presents the thermal modelling of such a system in order to assess the suitability of this approach. This work is complemented with the experimental work being carried out by the team in this subject, already with some published results. The model was validated with experimental data with a good correlation. Therefore, it was possible to demonstrate the potential of this system for wasted heat recovery.
\end{abstract}

\section{INTRODUCTION}

\section{A. Motivation}

The amount of energy wasted through the exhaust and the cooling system of a vehicle is roughly two times the mechanical power output of the engine [1]. The high temperature of the exhaust gases (up to $1000^{\circ} \mathrm{C}$ ) makes them potentially more apt for energy recovery than the lower temperature heat dissipated through the water-cooling system of the vehicle. The gains in efficiency for the vehicle could be relevant, even if a small percentage of this waste energy could be regenerated into electric power and used to charge the battery pack of a Hybrid or EREV vehicle, or even be used solely to prevent the actuation of a conventional vehicle's alternator.

Both Organic Rankine Cycle (ORC) [3] turbines and thermoelectric Generators (TEG) based on the Seebeck effect
[4][5][6] have been tested in heavy duty vehicles for exhaust heat recovery during the last decades. Nevertheless, both systems have limitations. ORCs have the highest recovery potential but they are still not viable for low duty engines [7], although some effort in low power applications has been made recently [8]. On the other hand, the efficiency of TEG systems has been improving significantly in the last few years due to the application of novel materials technology [9][10] but currently available systems still display a lower efficiency than ORCs. Nonetheless, for low power applications they currently stand as the only practical option since they are intrinsically scalable systems [7].

\section{B. Thermoelectric Modules}

It is known that the efficiency $(\eta)$ of thermoelectric conversion increases with the increase in temperature difference available. (1) models the efficiency of such devices, considering the hot side $\left(T_{H}\right)$ and cold side $\left(T_{C}\right)$ temperatures and the figure of merit $(\mathrm{ZT})$ of the materials, at a given mean temperature $T$ [11].

$$
\eta=\left(\frac{T_{H}-T_{C}}{T_{H}}\right)\left(\frac{\sqrt{1+Z T}-1}{\sqrt{1+Z T}+\frac{T_{C}}{T_{H}}}\right)
$$

Despite the increase of efficiency with temperature difference proposed with (1), the figure of merit (ZT) is not constant over the temperature. The ZT (which measures the ability of a material to generate energy from heat) is calculated considering the Seebeck coefficient $\alpha\left[\mathrm{VK}^{-1}\right]$ the electrical resistivity $\rho[\Omega \mathrm{m}]$ and the thermal conductivity $\lambda$ $\left[\mathrm{Wm}^{-1} \mathrm{~K}^{-1}\right]$ of the material, at a given temperature $T[\mathrm{~K}]$ as stated by (2).

$$
\mathrm{ZT}=\frac{\alpha^{2}}{\rho \cdot \lambda} T
$$

A candidate for a good thermoelectric material must have a high Seebeck coefficient (a large voltage is generated for a given temperature difference), a small resistivity (a large current could be drawn from the generator) and a small thermal conductivity (less heat will be used for the generator, increasing the efficiency). The ZT can also be calculated at a given temperature $\mathrm{T}$ for a thermoelectric module (instead of a single material), considering the voltage generated for each degree of temperature difference $V\left[\mathrm{VK}^{-1}\right]$, the electrical resistance $R i[\Omega]$ and the thermal conductance $K\left[\mathrm{WK}^{-1}\right]$ 


$$
\mathrm{ZT}=\frac{V^{2}}{R i . K} T
$$

The commercially available thermoelectric modules, made of Bismuth Telluride and Antimony Telluride have high efficiency for low temperature operation $\left(0-200{ }^{\circ} \mathrm{C}\right)$ but ZT decreases for temperature above $200{ }^{\circ} \mathrm{C}$, thus reducing efficiency. Moreover, mechanical stability of these modules often limits operation temperature to a maximum of $250^{\circ} \mathrm{C}$.

\section{B. Use of Heat Pipes for thermal control}

Although very simple in their working principle, HPs are of relatively recent use in industry [12]. A basic Heat Pipe consists of a sealed upright pipe containing a small portion of phase-changing fluid (see Figure 1 - left). The remainder of the inner volume of the pipe is occupied either by a mix of thermal fluid vapour and non-phase-changing gas (such as air) or solely by the former. Due to gravity, the fluid rests at the bottom of the pipe (the heat source region, or evaporator of the HP), where it will be heated and boiled under the action of the heat crossing the pipe walls through conduction. The vaporized fluid will eventually condense at the upper part of the pipe wall releasing its heat to the heat sink. Once condensed, the liquid droplets will fall back to the bottom of the pipe, completing the cycle and being ready to vaporize and condense over and over again.

A Heat Pipe will only start transferring heat from the hot source to the heat sink once the boiling temperature of the fluid has been achieved. This boiling temperature is not a static value but depends on the actual pressure of the fluid inside the HP (the pressure also changes during operation).

\section{VCHPS}

One way of controlling the phase changing temperature of a fluid would be to control its pressure. If the pressure could be kept constant at a specified value, then a certain HP operating temperature could be regulated. The use of an expansion tank attached to the top of the HP will enable this outcome (Figure 1 - right). Therefore, pressure would not build up during operation as it would in a standard HP and the boiling would not be hampered by an excessive increase of pressure. Such a system is called a Variable Conductance Heat Pipe (VCHP) and has been used in the current work.

The present work is being made in the scope of the development of a Range Extender for an electric vehicle. This particular Range Extender, based on the concept of overexpansion (Miller cycle in which the authors have expertise [2]), is to be used at a specified speed and load where its efficiency excels, producing relatively low power $(15 \mathrm{~kW})$. To harness the heat from its exhaust we should not need a $\mathrm{HP}$, as it would be possible to control the module temperature just by carefully choosing the conducting materials. However, our Range Extender has another point of operation, at a much higher power level $(40 \mathrm{~kW})$, therefore increasing the temperature level of the modules to values where they would fail. It was therefore decided to use a VCHP to avoid the problems arising from using two different power levels.

The comparison between the response of standard HPs and VCHPs for low and high thermal loads is outlined in Figure 1 (top). It is expected that with this system the HP will be able to work at a specified temperature. This will be made by adjusting the HP pressure to control the boiling temperature (the operating temperature of the VCHP) regulating it to the maximum allowable TEG temperature, thus optimizing the heat transfer rate of the system while protecting the system from over-heating.
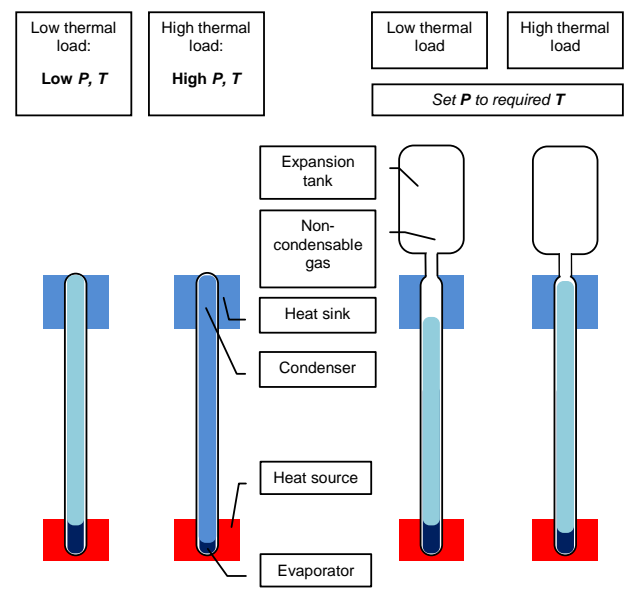

Standard HP $\underline{\mathrm{VCHP}}$

Figure 1 - Comparison of Standard and Variable Conductance Heat Pipes under variable thermal load

\section{B. Approach of the Present Work}

The present work tries to assess the potential of the combined use of VCHPs and commercially available, temperature limited TEGs for light duty vehicle exhaust systems. This study is still a proof-of-concept, having some simplifications: the evaporator is a brass block heated by a blowtorch flame as heat source, rather than using a finned structure with actual exhaust gases. A study with a more realistic automotive exhaust system will be made in future work. In the present work the hot side of the TEG modules is adjacent to the condenser while a cooling water circuit retrieves heat from the cold side of the modules in order to maximize the temperature difference across the thickness of the modules. To complement the analysis and optimize the design, the heat transfer and electric generation have been modelled and the predictions were compared against the experimental data.

The present paper is a follow-up of previous work done by the group on thermoelectric generators [13][14] both with and without heat pipe assist.

\section{THERMAL MODEL}

The modelling of the heat transfer within the system under study can be extremely useful to have a better understanding of the phenomena taking place and to further optimize the design of the system. The heat transfer between a hot and a cold source at temperatures $\bar{T}_{h o t}$ and $\bar{T}_{\text {cool }}$ can be modelled in a simplified way by accounting for the thermal resistances in the path of the heat flux, calculating the total equivalent thermal resistance, $R_{e q}$, and solving the equivalent thermal problem:

$$
\dot{Q}=\frac{\bar{T}_{h o t}-\bar{T}_{\text {cool }}}{R_{e q}}
$$

The system under study has two particularities that impose additional measures. 
The first specificity is that the temperature of vapour inside the heat pipe (here called the heat pipe operating temperature, $T_{H P}$ ) is fixed. Under steady state conditions this temperature corresponds to the boiling temperature of the working fluid for the inner pressure of the HP. In the system under study this pressure is regulated a priori and kept constant throughout the test thanks to a large expansion tank that has been added. Under these conditions, thermal calculations have to be carried out separately in two different sections of the heat flux path: sector 1, from the exhaust gas up to the vaporized fluid and sector 2, from there up to the water cooling system (Figure 2).

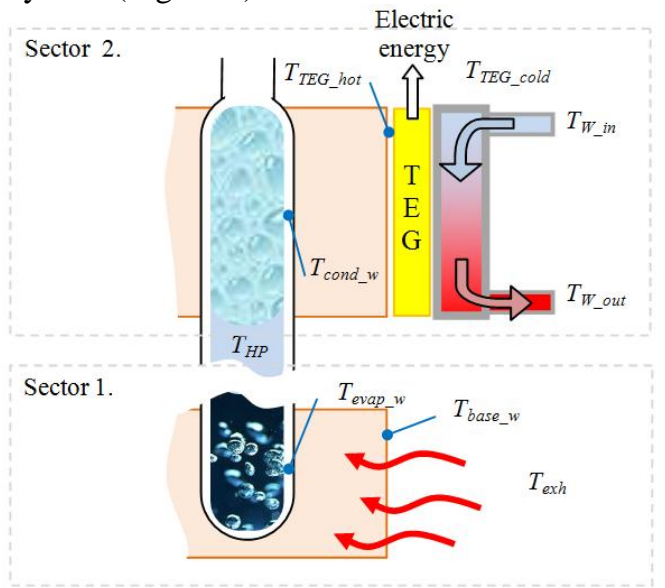

Figure 2 - Outline of the TEG generator and relevant temperatures

The thermal power across sectors 1 and 2 is calculated with (4) using the average temperatures of the extremities $\left(\bar{T}_{\text {hot }}\right.$, $\bar{T}_{\text {cool }}$ ) and the equivalent thermal resistance in-between. At steady state the power crossing each sector must converge to the same value since no thermal losses are considered along the heat flux path. This means that the thermal power across the system will be limited by the smaller of the two. In physical terms this means that under steady state conditions the mass conservation condition imposes that both evaporation and condensation rates stabilize around the same value. This problem is treated further ahead.

This system has a second specificity, since between the thermal source and sink there is some energy which exits through TEG module under the form of electricity. This creates further complexity in the model, but (4) can still be used, as explained further along this document.

The thermal resistance to a heat flux depends on the heat transfer coefficient $h$, and the area normal to the heat flux $A$ :

$$
R=1 / h A
$$

For conduction across a body with section $A$, thickness $t$, and thermal conductivity $k$, the heat transfer coefficient is $h=k / t$. If there is a non-uniform section/thickness across the heat flux path, then a shape factor must be used. This factor is a thermal equivalent of the $A / t$ relation, $(A / t)_{e q}$. For convection, $h$ can be estimated from the Nusselt number, $N u=\frac{h D}{k}$, for the particular application, with $D$ being the characteristic length.

The basic heat flux path within the system is the following (see Figure 2): a) The hotter exhaust gas supplies heat to the outer walls of the HP evaporator through convection. The expression used to estimate the average Nusselt number for cross flow over a cylinder is the one proposed by Churchill and Bernstein [15]:

$$
N u=0.3+\frac{0.62 \operatorname{Re}^{1 / 2} \operatorname{Pr}^{1 / 3}}{\left[1+\left(\frac{0.4}{P r}\right)^{2 / 3}\right]^{1 / 4}} \cdot\left[1+\left(\frac{R e}{2.82 \cdot 10^{5}}\right)^{5 / 8}\right]^{4 / 5}
$$

b) The heat crosses the evaporator's walls by conduction, reaching the inner HP walls. The shape factor for this geometry (concentric cylindrical surfaces) is [15]:

$$
(A / t)_{e q}=\frac{2 \pi L}{\ln \left({ }^{D_{\text {out }} / D_{\text {in }}}\right)}
$$

c) At the wetted section of the evaporator the heat is transmitted from the wall to the working fluid, vaporizing it. The Rohsenow expression for nucleate boiling has been used [16]:

$$
N u=\frac{1}{C_{s f}} \operatorname{Re}^{(1-x)} \operatorname{Pr}^{-y}
$$

Where $C_{s f}$ is a constant dependent on the interface $(0.013$ for a water-copper interface), $x$ and $y$ are constants ( 0.33 and 0.7 , respectively, for most fluids) and $R e$ is the Reynolds number which is representative of the velocity with which the liquid flows towards the surface to replace that which has been vaporised [16]:

$$
R e=\frac{\rho_{l v D}}{\mu} ; v=\frac{\dot{q}}{\rho_{l} H_{L}} ; d=\left[\frac{\sigma_{l}}{g\left(\rho_{l}-\rho_{v}\right)}\right]^{0.5}
$$

Where $H_{L}$ is the enthalpy of condensation, $\sigma_{l}$ is the surface tension and $\dot{q}$ is the thermal power per unit area, which has to be estimated initially and then iterated until convergence.

Since the evaporator is only partially wetted, a small portion of the heat flux will also cross the evaporator walls through its dry region. This heat will be first transmitted to the vapour by convection. To estimate the convection coefficient, the Dittus-Boulter expression for turbulent flow inside a smooth pipe has been used to obtain the Nusselt number [15]:

$$
N u=0.023 \cdot R e^{0.8} \cdot \operatorname{Pr}^{0.4}
$$

The Reynolds number has been estimated with the vapour volumetric flow rate across the section of the evaporator resulting from the vapour production rate (obtained from the evaporative power estimation and the enthalpy of vaporization) and a multiplying factor of 5 in order to account for the intrinsic disruptive nature of boiled vapour flow.

Due to the high degree of mixing, agitation and turbulence which is typical of boiling processes, it seems reasonable to assume that in the end the heat crossing the dry walls of the evaporator and transmitted to vapour will also be directed to boiling. Therefore, the heat transfer between the outer wall of the evaporator and the vapour exiting the evaporator towards the condenser can be considered to occur in parallel along two paths crossing the wet and dry portions of the evaporator, respectively. The equivalent thermal resistance for this process is then:

$$
1 / R_{\text {wet }+ \text { dry }}=1 / R_{\text {wet }}+1 / R_{\text {dry }}
$$

Where $R_{\text {wet }}$ and $R_{\text {dry }}$ are the thermal resistances for the heat fluxes through the wet and the dry walls of the evaporator, respectively: 


$$
\left\{\begin{array}{l}
R_{\text {wet }}=\frac{1}{k_{\text {base }}\left({ }^{A} / t\right)_{\text {eq }}}+\frac{1}{h_{\text {evap }} A_{\text {wet }}} \\
R_{\text {dry }}=\frac{1}{k_{\text {base }}\left({ }^{A} /\right)_{\text {eq }}}+\frac{1}{h_{\text {vapor }} A_{\text {dry }}}
\end{array}\right.
$$

d) By diffusion and buoyancy the vaporized working fluid moves towards the upper part of the HP. This mass/energy transfer resistance has not been considered in the calculations as it should be much lower than other terms. As the vapour reaches the heat sink region of the HP it condenses, releasing the latent heat to the walls. According to the Nusselt theory described in [16], the average heat transfer coefficient for film condensation over a condenser of active length Lc is the following:

$$
h_{\text {cond }}=0.943\left[\frac{H_{L} \rho_{l}^{2} g k_{l}^{3}}{L_{c} \mu_{l}\left(T_{s}-T_{\text {cond_w }}\right)}\right]^{0.25}
$$

Where, Ts is the temperature of the liquid film surface (considered as being the saturation temperature) and Tcond_w being the condenser wall temperature;

e) Heat crosses the walls of the condenser by conduction, reaching the hot side of the modules. The shape factor for conduction between an inner cylindrical surface of diameter $\mathrm{D}$ and an outer square prism of width a and length $\mathrm{L}$ is [17]:

$$
(A / t)_{e q}=\frac{2 \pi L}{\ln \left(\frac{1.08 a}{D}\right)}
$$

f) Heat crosses the modules by conduction, while a fraction of it (the TEG efficiency, $\varepsilon$ ) is converted into electric energy due to the Seebeck effect. Therefore, downstream of the TEGs, the thermal power $\dot{Q}$ will reduce to $\dot{Q}(1-\varepsilon)$. It is possible to incorporate this heat sink into temperature calculations making use of the general analytical solution for the one-dimensional temperature field across a plane wall with a uniform heat source/sink [18]:

$$
T(x)=\dot{q} / 2 k^{2}+C_{1} x+C_{2}
$$

Where $\mathrm{x}$ is the coordinate along the thickness of the TEG and $\dot{\mathrm{q}}$ is the thermal power generation per unit volume, which in the present case becomes:

$$
\dot{q}=-\frac{\dot{Q} \varepsilon}{A_{T E G} t_{T E G}}
$$

Now the constants of integration $\mathrm{C} 1$ and $\mathrm{C} 2$ can be determined from two different boundary conditions, for instance, the temperature and the heat transfer rate at the hot face. Considering this face as the origin $(\mathrm{x}=0)$, the following applies:

$$
\left\{\begin{array}{c}
T(0)=T_{T E G_{h o t}} \rightarrow C_{2}=T_{T E G_{h o t}} \\
-k_{T E G} A_{T E G} \frac{d T(0)}{d x}=\dot{Q} \rightarrow C_{1}=-\frac{\dot{Q}}{k_{T E G} A_{T E G}}
\end{array}\right.
$$

Considering that $\mathrm{T}_{\mathrm{TEG}_{\text {cool }}}=\mathrm{T}(\mathrm{t})$ the temperature difference across the TEG faces can be obtained:

$$
T_{T E G_{\text {hot }}}-T_{T E G_{\text {cool }}}=\frac{\dot{Q}}{R_{T E G}} ; R_{T E G}=\frac{t}{k_{T E G} A_{T E G}}\left(1-\frac{\varepsilon}{2}\right)
$$

g) The remaining thermal power $\dot{Q}(1-\varepsilon)$ crosses the walls of the cooling ducts by conduction and is transmitted to the coolant by convection, modelled by the Sieder and Tate expression for developing laminar flow in circular pipes [15]:

$$
N u=1.86\left(\frac{\operatorname{RePr} D_{h}}{L}\right)^{1 / 3}\left(\frac{\mu_{b}}{\mu_{s}}\right)^{0.14}
$$

This expression can be adapted for non-circular ducts by using the equivalent hydraulic diameter (four times the cross section divided by the perimeter), Dh.

It should be noted that contact thermal resistances have been neglected, as thermal grease was used in the experimental prototype.

Once the individual thermal resistances have been estimated, the total equivalent thermal resistance for each calculation sector, $R_{e q 1}$ and $R_{e q 2}$ is can be obtained from the individual resistances:

$\left\{\begin{array}{c}R_{\text {eq } 1}=\frac{1}{h_{\text {exh } A_{\text {base }}}}+R_{\text {wet }}+\text { dry } \\ R_{\text {eq } 2}=\frac{1}{h_{\text {cond }} A_{\text {cond }}}+\frac{1}{k_{c_{-} \text {block }}\left({ }^{A} / t\right)_{e q}}+R_{T E G}+\frac{t_{\text {duct }}(1-\varepsilon)}{k_{\text {duct }} A_{\text {duct }}}+\frac{1-\varepsilon}{h_{w} A_{w}}\end{array}\right.$

From this, the thermal powers at sectors 1 and 2 can be calculated with (4). Now, as said above, the thermal powers at the side of the evaporator and the condenser must be equal at steady state, since the sector with lower power is acting as a bottleneck for the sector with higher power. For instance, if the bottleneck is at the side of condensation this means that less and less liquid will fall back into the evaporator. This will cause a reduction of the wetted surface that receives the heat from the wall, decreasing the evaporation rate. Eventually the evaporation rate will be levelled with the condensation rate reaching equilibrium. Therefore, an iteration of the liquid height (and therefore the heat exchange area) must be performed until the evaporative power equals the condensation power. Similarly, if the bottleneck is located at the evaporation side, the active heat exchange area of the condenser has to be reduced iteratively until both evaporation and condensation powers are levelled. Other iterative calculations must be performed within the heat transfer algorithm. For instance, the average temperature of the cooling fluid, $\bar{T}_{W}$, that enters into the calculations, depends on the actual thermal power reaching the water, which is only known once thermal resistances have been estimated:

$$
\bar{T}_{W}=\frac{T_{W_{\text {in }}}+T_{W_{-} \text {out }}}{2} ; T_{W_{-} \text {out }}=T_{W_{-} \text {in }}+\frac{\dot{Q}}{\dot{m}_{W} c_{p}}
$$

Therefore, $\bar{T}_{W}$ has to be updated after the power has been estimated.

The intermediate temperatures are easily obtained in cascade using (4) and the HP power calculated previously. Once the temperatures at the cold and hot sides of the TEG (TEGhot and TEGcold) have been determined, it is possible to estimate the electric power generated by the system as detailed in the next section.

The present model was successfully implemented in a spreadsheet, including the physical properties such as thermal conductivity, density, specific heat, viscosity and Prandtl number. This model will be vital for designing the final HP, as the right areas for evaporation and condensation will have to be right for avoiding the referred bottlenecks.

\section{ELECTRIC MODEL}

The thermoelectric modules produce a voltage $\left(V_{O}\right)$ that is proportional to the temperature difference between the hot and the cold plates of each module (22). In it, $\alpha$ is the Seebeck coefficient of the thermoelectric module, $n$ is the number of thermoelectric modules and $T_{H}$ and $T_{C}$ are the temperatures at the hot side and cold side of the modules, 
respectively. The mean Seebeck coefficient is considered, since the Seebeck coefficient is not constant over the working temperature range. All modules are connected electrically in series, thus the total voltage generated $\left(V_{O}\right)$ and the total internal resistance $\left(R_{i}\right)$ are the sum of the voltage produced by each module and the internal resistance of each module, respectively

$$
V_{o}=n \alpha\left(T_{H}-T_{C}\right)
$$

The maximum power transfer from the modules to the load $\left(P_{O}\right)$ occurs when the load resistance $\left(R_{L}\right)$ equals the sum of internal resistance of the modules $\left(R_{i}\right.$ is the electrical resistance of each module and $n \cdot R_{i}$ is the total resistance, when the modules are connected in series). A mean value is used since the resistance is not constant over the working temperature range.

$$
P_{o}=\frac{\left(n \alpha\left(T_{H}-T_{C}\right)\right)^{2}}{4 n R_{i}}
$$

The voltage and current for different electrical loads, were measured, with five temperature differences $(\Delta T=\{75,47$, $\left.30,13,8\}^{\circ} \mathrm{C}\right)$. Despite the small temperature difference, these results, plotted on Figure 3, demonstrate the ability to produce electricity from a temperature gradient. Moreover, the almost linear $V$-I plot, indicate that internal resistance of the generator $\left(R_{i}\right)$ is almost constant over the load plot, and almost constant over the temperature.

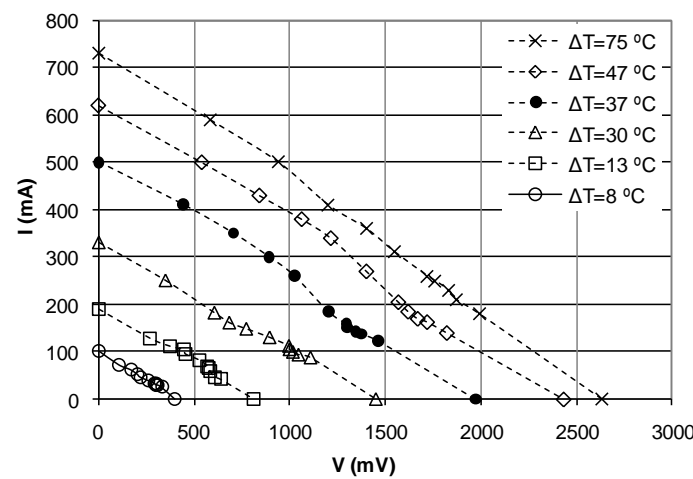

Figure 3 - Voltage (V) and current (I) output in experimental characterization of thermoelectric modules, for temperature differences between hot and cold face $(\Delta \mathrm{T})$ from $8{ }^{\circ} \mathrm{C}$ to $75^{\circ} \mathrm{C}$.

This linear behaviour simplifies the implementation of simple Maximum-Power-Point-Tracking (MPPT) electronic circuit to use the available power in an efficient manner, charging the vehicle batteries at full available power. At any operation point $(V, I)$, at any temperature, the optimum operation point $\left(V_{O P T}, I_{O P T}\right)$ can be easily calculated, ever using analogue circuits, using (24), since the internal resistance $(R i)$ is constant and previously known.

$$
V_{O P T}=1 / 2\left(V+R_{i} \times I\right)
$$

With the VOPT calculated, the duty cycle (D) of a DC-DC step down converter circuit can be calculated just measuring the output voltage (V) and current (I) of the generator ( $\mathrm{G}$ is the gain of the control loop):

$$
D=G\left(V-V_{\text {OPT }}\right)=1 / 2\left(V-R_{i} \times I\right)
$$

This is implemented with circuit of figure 4. TEG is the thermoelectric generator, I Sense is a current sensor and $\mathrm{VCO} / \mathrm{D}$ is a square-wave oscillator with duty cycle controlled by voltage.

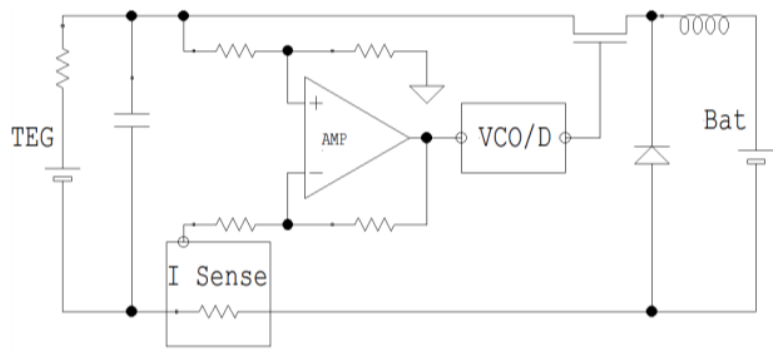

Figure 4 - Simple MPPT step-down DC-DC converter circuit for a thermoelectric generator.

\section{TEST RIG AND EXPERIMENTAL PROCEDURE}

The experimental prototype (Figure 5) consists of a $22 \mathrm{~mm}$ bore copper pipe, used as heat pipe. Its lower part includes a solid wide diameter brass base where heat is supplied by a blowtorch. Its interior serves as the evaporator of the heat pipe. The upper part of the HP (condenser) is formed by a square bloc of brass, where the Seebeck modules are attached. Eight modules cover the whole of the 4 faces. On the external side of these modules 4 aluminium flat tubes are used as the cold sink of the modules. Inside these tubes cold water is forced at a specified rate.

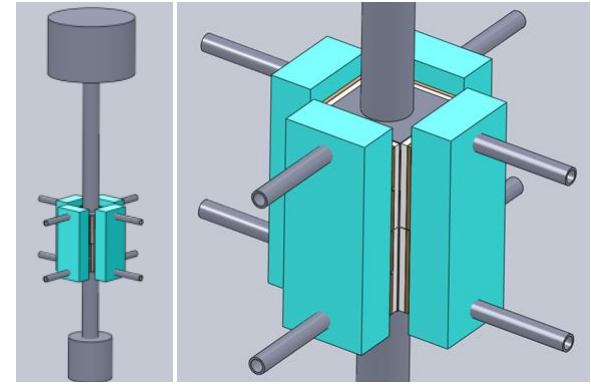

Figure 5 - Experimental installation with detail of module arrangement and cooling of the cold face of the modules.

The top of the HP is connected to a large cylinder vessel $(20 \mathrm{~L})$. The purpose is to maintain the HP pressure at the specified value, therefore setting the required temperature of phase change of the fluid (water).

Thermocouples were placed at various locations to measure the temperature and heat fluxes throughout the prototype. Care was taken to minimize the effect of contact thermal resistance, using thermal grease. It was also possible to assess the maximum potential for the supplied heat by looking at the temperatures of the pipe connecting the HP to the large vessel. If some of these temperatures were bellow the boiling temperature for the set pressure and others were the boiling temperatures, we could be sure that the condenser was working fully and there was no vapour passing to the large vessel. That was the setting for steady-state at maximum heat flux.

The aluminium flat ducts were connected in series and the water flow was set at a level where the temperature difference was enough for proper heat calculation, but was high enough for keeping the modules at the lowest possible temperature. A connection is series was preferred to parallel as the water 
velocity would be 4 times higher (in each tube) for the same total temperature difference, therefore enhancing the heat transfer coefficients. This is detailed in Figure 5.

The thermoelectric modules used in the generator (TEC112708 from Thermoelectric Supplier) were connected electrically in series. These modules, with dimensions of $40 \times 40 \times 3.6 \mathrm{~mm}^{3}$, can support temperatures up to $238^{\circ} \mathrm{C}$.

In half of the modules, the open circuit voltage (generator without load) was measured. In the other half, output voltage was measured with a known $\mathrm{Ohm}$ resistive load connected at the output. With this setup, considering the open circuit voltage and voltage at a given load, internal resistance of the module and maximum available power (obtained with maximum power point tracking hardware at a matched output load) was calculated, considering a linear resistive internal impedance on the generator. Efficiency was calculated considering the maximum available electrical power (at a matched load) and the thermal power through the module.

\section{RESULTS AND DISCUSSION}

One test can be seen in Figure 6, where the various temperatures and heat fluxes were plotted against time. Please note that, while thermocouples T1 and T2 reached the boiling temperature value, T3 and T4 remained much cooler. Since these thermocouples are located between the HP and the expansion tank (T1 closest to HP, T4 closest to tank), this indicates that the vapour did not reach the upper part of the tube connecting to the large vessel, but it filled completely the condensation volume.

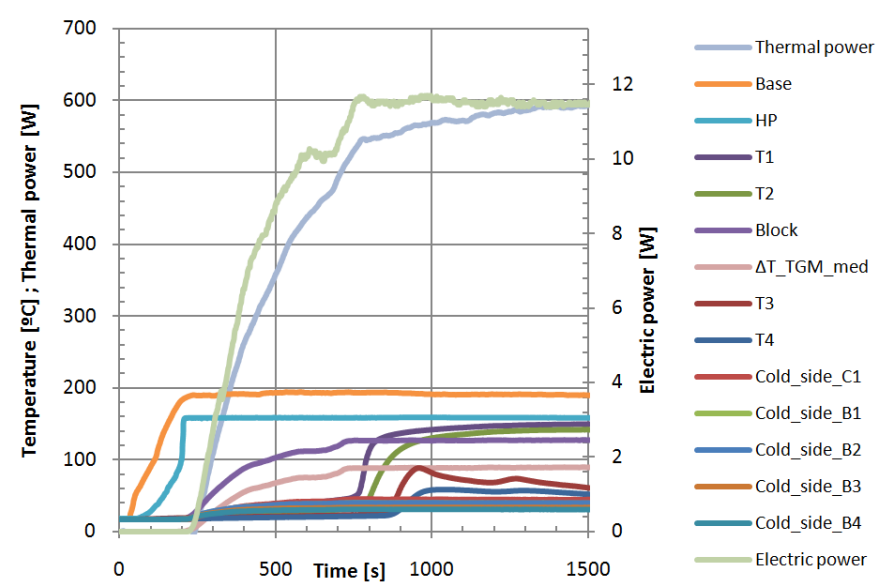

Figure 6 - Temperature and thermal and electric power for 6.2 bar

If too much power is supplied to the evaporator of the HP, then the mass flow rate of vapour could not be fully condensed on the condenser, and it will enter the tank. Eventually, all the liquid might disappear from the evaporator and the temperature of the base would increase, signalling the lack of heat removal at the evaporator. This happened in one test, for low pressure (1 bar), as seen in Figure 7 (note that thermocouples T1 thru T4 all display temperatures around the boiling value).

\section{Comparison of model and experimental results}

Using the thermal model presented in (1) and (4) thru (21) and the electric model represented in (2), (3) and (22) thru
(25) it was possible to compare the experimental results with the model predictions (Figure 8).

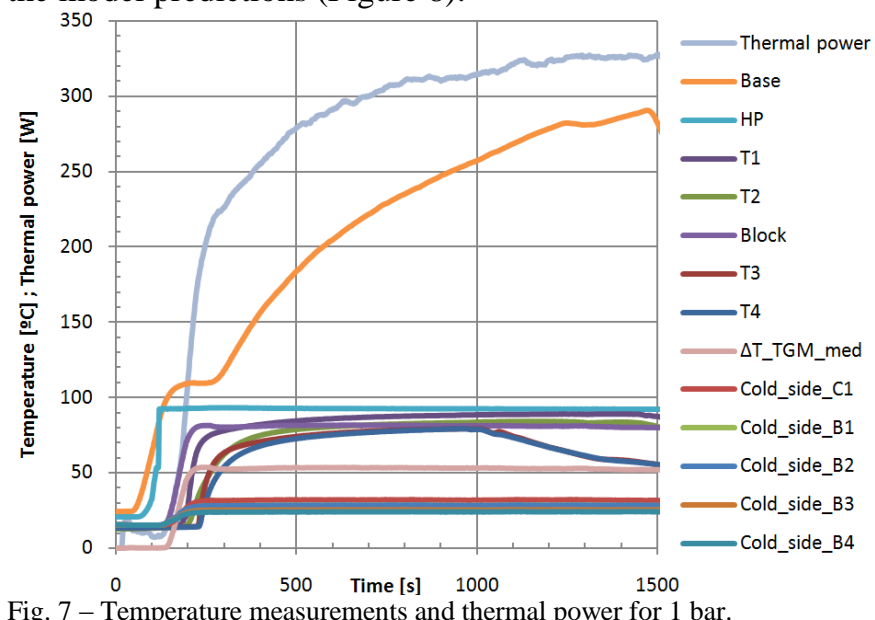

The measured thermal power shows the trend predicted by the model, but some of the measured values are somewhat higher than predicted. This might indicate that the condensation power has been slightly under-predicted. This might be due to the intrinsic limitations of condensation modelling, namely having considered pure "film condensation". Nevertheless, the maximum difference was around $8 \%$ for the $158^{\circ} \mathrm{C}$ test $(6.2$ bar test), which, given the complexity of condensation phenomena, can be taken as acceptable.

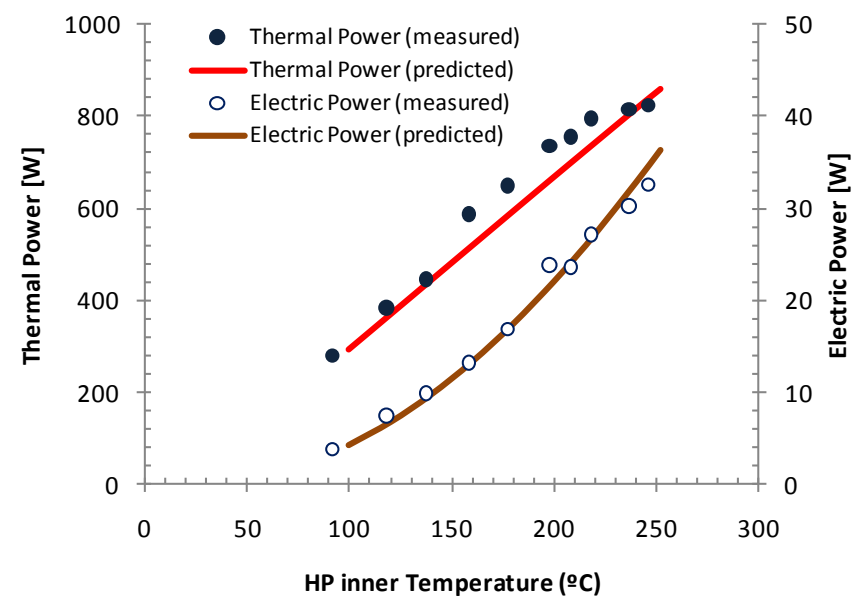

Figure 8 - Thermal power vs HP temperature

Figure 8 also displays the electrical power produced by the modules as a function of HP temperature. Here the correlation between theory and experiment is rather good, although in the highest temperature tests the electric power output has been slightly over-predicted. But again these are very small differences that fall below the uncertainty margin.

The various temperatures recorded at the experimental rig for the 6.2 bar test (recall Fig. 6) can be seen in Figure 9 (red dots) compared against the temperatures predicted by the model (blue line/dots). In Sector 1 (evaporator region) the correlation between theory and experiment is notable. In Sector 2 (condenser/TEG/cooling) the prediction of the temperature of the hot wall of the TEG is somewhat lower than measured. This fact is in line with the underprediction of the thermal power already discussed. This was the test with 
the largest differences between theory and experiment. Nevertheless, the maximum difference was below $14 \%$.

As a final remark it can be concluded that, despite the slight differences found between theory and experiment, the proposed model is sufficiently validated and is suitable for predicting the thermal behaviour of Heat-Pipe-assisted thermoelectric generators.

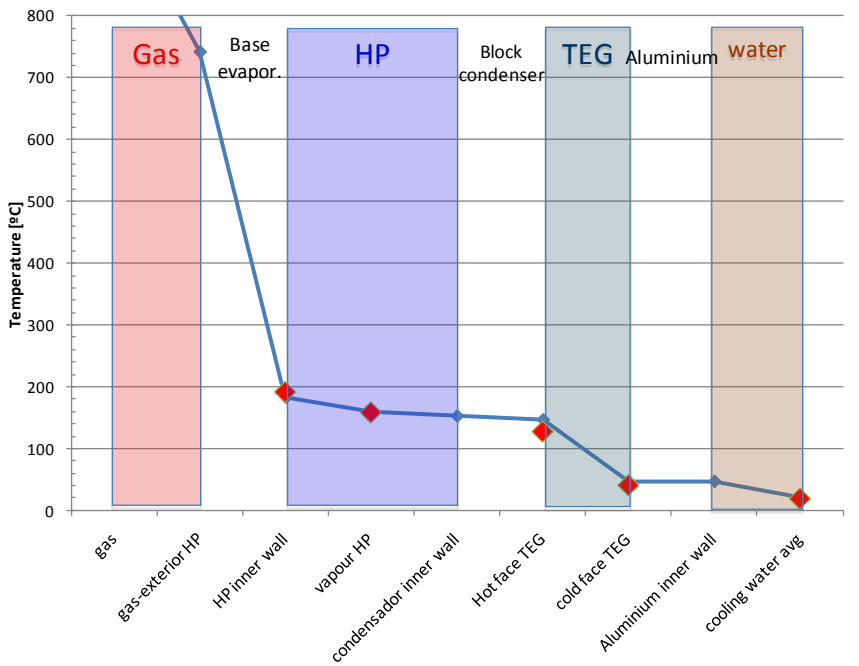

Figure 9 - Temperature within the installation (model and measurements)

\section{CONCLUSIONS}

A model for predicting the operation of an energy recovery system that converts thermal wasted heat (from exhaust gases) into electricity using thermoelectric generators and heat pipes was developed and presented. This thermal model covers the various types of thermal interactions within the experimental system.

Globally, the model predictions, made for a wide range of HP pressure (from 1 to 40 bar) were satisfactory, with maximum differences between theory and experiment below $10 \%$ for thermal power and within the uncertainty margin for electric output. Therefore, the model can be considered to be sufficiently validated and apt for aiding in the design of HeatPipe-assisted thermoelectric generators for engine exhaust heat recovery applications, namely for variable thermal load applications such as a range extender unit under development by the team.

\section{ACKNOWLEDGMENTS}

This research project was supported by MIT-Pt/EDAMSMS/0030/2008 and FCT. F. Brito acknowledges FCT and the MIT-Portugal programme for the post-doctoral grant SFRH/BPD/51048/2010.

\section{REFERENCES}

[1] Heywood J., Internal Combustion Engine Fundamentals, McGraw Hill, 1988.

[2] Ribeiro B., Martins, J., "Direct Comparison of an Engine Working under Otto, Miller and Diesel cycles: Thermodynamic Analysis and Real Engine Performance", SAE Technical Paper Series, n² 2007-010261, 2007;

[3] Hung T. C., T. Y. Shai T. Y., Wang S. K. "A review of organic rankine cycles (ORCs) for the recovery of low-grade waste heat", Energy, Elsevier, 22-7, pp. 661-667, 1997
[4] Birkholz, U., Grob, U. Stohrer and K. Voss, "Conversion of Waste Exhaust Heat in Automobile using FeSi2 Thermoelements". Proc. 7th International Conference on Thermoelectric Energy Conversion. 1988, Arlington, USA, pp. 124-128, 1988

[5] Bass J. C., Elsner N. B. and Leavitt F. A. "Performance of the $1 \mathrm{~kW}$ Thermoelectric Generator for Diesel Engines" International Conference on Thermoelectrics, 1994, Kansas City, Kansas, USA, 1994.

[6] Kushch A., Karri M. A., Helenbrook B. T. and Richter Clayton J., "The Effects of an Exhaust Thermoelectric Generator of a GM Sierra Pickup Truck." Proceedings of Diesel Engine Emission Reduction (DEER) conference, 2004, Coronado, California, USA, 2004

[7] Vining C.B. "An inconvenient truth about thermoelectrics", Nature Materials, 8, February 2009;

[8] Zhang X., Zeng K., Bai S., Zhang Y., He M. "Exhaust Recovery of Vehicle Gasoline Engine Based on Organic Rankine Cycle", SAE paper 2011-01-1339, SAE International, 2011

[9] Hogan T.P. et al, "Nanostructured Thermoelectric Materials and HighEfficiency Power-Generation Modules", Journal of Electronic Materials, Vol. 36, No. 7, 2007

[10] Pei Y., Shi X., LaLonde A., Wang H., Chen L., Snyder J. "Convergence of electronic bands for high performance bulk thermoelectric", Nature, 473 (05 May 2011), pp. 66-69, 2011

[11] Rowe D. M, "Thermoelectrics, an environmentally-friendly source of electrical power", Renewable Energy, Volume 16, Issues 1-4, Pages 1251-1256, 1999

[12] Reay D., Kew P. "Heat Pipes: Theory, Design and Applications", BH, 2006

[13] Gonçalves L.M., Martins J., Antunes J., Rocha R. and, Brito F. P.”Heat-Pipe Assisted Thermoelectric Generators for Exhaust Gas Applications", ASME 2010 International Mechanical Engineering Congress \& Exposition, November, 12-18, 2010, Vancouver, British Columbia, Canada, 2010.

[14] Martins J., Brito F.P., Goncalves L.M., Antunes J. "Thermoelectric Exhaust Energy Recovery with Temperature Control through Heat Pipes", SAE paper 2011-01-0315, SAE International Publ., 2011;

[15] Çengel Y.A., Turner R.H. "Fundamentals of Thermal-Fluid Sciences", McGraw-Hill, International Edition, 2001

[16] Reay D., Kew P. "Heat Pipes: Theory, Design and Applications", BH, 2006

[17] Martínez I. "Thermodynamics, Heat conduction modelling: Conduction shape factor (steady state)", available online (accessed May 2011); http://webserver.dmt.upm.es/ isidoro/bk3/c11/Heat\%20conduction.ht m\# Toc254106124, 2011

[18] Incropera F.P., deWitt D.P., "Fundamentals of Heat and Mass Transfer", 3rd Ed., Wiley, 1990 